\title{
ARTICLE Inhibitory effects of lappaconitine on the neuronal isoforms of voltage-gated sodium channels
}

\author{
Yan-fen $\mathrm{Li}^{1,2}$, Yue-ming Zheng ${ }^{2,3}$, Yong Yu ${ }^{4}$, Yong Gan ${ }^{2,3}$ and Zhao-bing Gao ${ }^{2,3}$
}

\begin{abstract}
Lappaconitine (LA) has been widely used for postoperative and cancer pain control. LA exhibits excellent analgesic activity with a longer effective time than common local anesthetics such as tetracaine and bupivacaine. However, the mechanisms underlying the featured analgesic activity of LA remain largely unknown. Here, we report that LA is an inhibitor of voltage-gated sodium channel 1.7 (Nav1.7) stably expressed in human embryonic kidney (HEK293) cells. LA inhibited Nav1.7 in a voltage-dependent manner with an $\mathrm{IC}_{50}$ value (with $95 \%$ confidence limits) of $27.67(15.68-39.66) \mu \mathrm{mol} / \mathrm{L}$ when the cell was clamped at $-70 \mathrm{mV}$. In comparison with the quick and reversible inhibition of Nav1.7 by tetracaine and bupivacaine, the inhibitory effect of LA was rather slow and irreversible. It took more than $10 \mathrm{~min}$ to achieve steady-state inhibition when LA ( $300 \mu \mathrm{mol} / \mathrm{L})$ was administered. Unlike tetracaine and bupivacaine, LA affected neither the voltage-dependent activation nor the inactivation of the channels. Five residues in domain III and domain IV have been reported to be critical for the effects of the two local anesthetics on Nav channels. But our mutant study revealed that only two residues (F1737, N1742) located in domain IV were necessary for the inhibitory activity of LA. The slow onset, irreversibility, and lack of influence on channel activation and inactivation accompanied with the different molecular determinants suggest that LA may inhibit Nav1.7 channels in a manner different from local anesthetics. These results may help to understand the featured analgesic activity of LA, thus benefiting its application in the clinic and future drug development.
\end{abstract}

keywords: voltage-gated sodium channel 1.7; analgesics; lappaconitine; tetracaine; bupivacaine

Acta Pharmacologica Sinica (2019) 40:451-459; https://doi.org/10.1038/s41401-018-0067-x

\section{INTRODUCTION}

Lappaconitine (LA) is a diterpene alkaloid extracted from Aconitum species and widely employed in Chinese and Japanese medicine mainly for analgesic indications [1]. Compared to other aconitum alkaloids, LA exhibits stronger antinociceptive efficacy and less toxicity [2]. LA can be administered through intravenous, intramuscular, intrathecal, and oral routes for analgesic treatment, and LA adhesive patch is also widely used in the clinic [3]. The analgesic activities of LA are generally approximately seven times greater than those of phenazone, a commonly used non-steroidal anti-inflammatory drug and are almost equipotent to pethidine and tramadol [4-6]. The analgesia maintenance time for a single epidural application of LA is approximately $8 \mathrm{~h}$, which is much longer than that for local anesthetics such as tetracaine and bupivacaine [7, 8]. Notably, the outcome of patients administered LA is more satisfactory than that of those administered opioids, and the incidence of urinary retention, nausea, and other side reactions is lower $[9,10]$. These advantages can be partly attributed to the fact that the analgesic activities of LA are not associated with the opioid system, as revealed by the findings that the actions were not antagonized by naloxone [11, 12]. Moreover, clinical applications have demonstrated that the combination of LA and opioids produces a significant reduction in effective dosage and an enhancement in analgesic efficacy $[7,13]$.
Currently, LA alone or in combination with other opioids is recommended as a therapeutic choice for moderate and severe postoperative and cancer pain in China [3].

Several studies in rodents have found that central noradrenergic and serotonergic systems might be involved in the antinociceptive effects of LA. A reduction in the concentration of noradrenaline and damage to the serotonergic neurons in the central nervous system suppressed the analgesic activity of LA [14, 15]. Another study using a chronic constriction injury rat model argued that LA may reduce the expression of the $\mathrm{P} 2 \mathrm{X} 3$ receptor in rat dorsal root ganglion neurons [16]. In rat brain slices, LA was found to suppress the epileptiform activities of neurons [17-19]. Consistent with the observed suppression of neuronal excitability in brain slices, LA exhibited inhibitory effects on depolarizationelicited sodium currents in rat hippocampal and trigeminal ganglion neurons $[20,21]$, which suggested that inhibition of voltage-gated sodium (Nav) channels may contribute to the analgesic effects of LA. The Nav channel family contains nine members, which are essential for electrogenesis in excitable cells and are key targets for local anesthetics, antiepileptics, and antiarrhythmics [22]. Interestingly, spinal cord transection at T9-T11 was unable to abolish the LA-induced antinociception in mice subcutaneously pretreated with $L A$, which suggested that peripheral analgesic targets, perhaps Nav channels, are involved in

\footnotetext{
${ }^{1}$ Shanghai Key Laboratory of Bio-Energy Crops, School of Life Sciences, Shanghai University, Shanghai 200444, China; ${ }^{2}$ CAS Key Laboratory of Receptor Research, State Key Laboratory of Drug Research, Shanghai Institute of Materia Medica, Chinese Academy of Sciences, Shanghai 201203, China; ${ }^{3}$ University of Chinese Academy of Sciences, Beijing 100049, China and ${ }^{4}$ Department of Neurosurgery, Zhongshan Hospital, Fudan University, Shanghai 200032, China

Correspondence: Yong Gan (ygan@simm.ac.cn) or Zhao-bing Gao (zbgao@simm.ac.cn)
}

Received: 10 March 2018 Accepted: 17 May 2018

Published online: 10 July 2018 


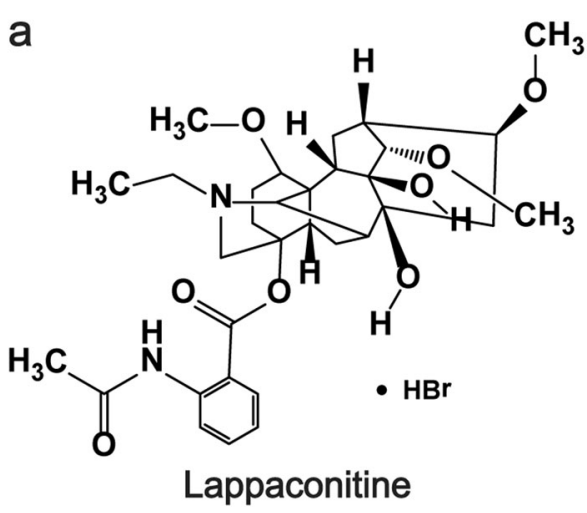<smiles>CCCN1CCCCC1C(=O)Nc1c(C)cccc1C</smiles>

\section{Bupivacaine}

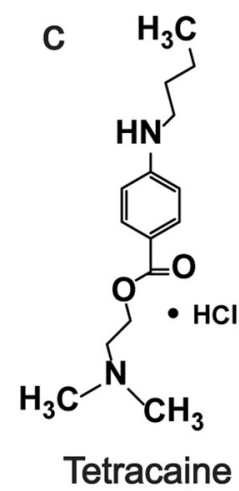

Fig. 1 The structures of lappaconitine (LA), bupivacaine, and tetracaine

the analgesic effect of LA [15]. Consistently, an LA adhesive patch has also been widely used in the clinic for pain relief [23, 24].

The Nav1.7 channel, the seventh member of the Nav family, has been proposed to be a promising analgesic target [25]. Compared to other Nav isoforms, Nav1.7 is preferentially distributed in nociceptive dorsal root ganglion neurons and sympathetic ganglion neurons [25, 26]. Nav1.7 is composed of a single polypeptide with four homologous domains (DI-DIV), with each domain containing six transmembrane segments (S1-S6), in which $\mathrm{S} 1-\mathrm{S} 4$ form the voltage-sensing domain (VSD) and S5-S6 form the pore region [25]. The ability of Nav1.7 to amplify small subthreshold stimuli increases the probability that neurons reach their threshold for firing action potentials, thereby acting as an important determinant of threshold in nociceptors $[25,26]$. The crucial role of Nav1.7 in pain signaling has been highlighted by several inherited mutations in which gain-of-function mutations cause congenital pain and loss-of-function mutations lead to a congenital painlessness [26]. Currently, several Nav1.7 channel selective inhibitors, including an antibody, toxins, and small molecules, have been developed [27]. Clinical studies showed that inhibition of Nav1.7 channel by these inhibitors could attenuate pain in patients with inherited erythromelalgia $[25,28]$. In the consideration of the pivotal role of Nav1.7 in pain, in the present study, we set out to characterize the effects of LA on the Nav1.7 channel using electrophysiological methods and provide evidence that LA is an inhibitor of Nav1.7.

\section{MATERIALS AND METHODS}

Materials

LA was purchased from Dahe Pharmaceutical Co. (Shaanxi, China). The purity of the synthetic LA was $\geq 98 \%$ as assessed by HPLC. The compound was dissolved and stored in dimethyl sulfoxide (DMSO) to produce $20 \mathrm{mmol} / \mathrm{L}$ stock solutions that were then diluted in the bath solution to obtain the final concentrations. The final concentration of DMSO $(\leq 0.5 \%)$ was tested and verified to have no observable effect on sodium currents. Bupivacaine and tetracaine were purchased from Sigma-Aldrich (St. Louis, MO, USA). GX-936 was synthesized in the laboratory of Prof. Fa-jun Nan (Shanghai Institute of Materia Medica, Chinese Academy of Sciences, Shanghai, China). TTX was purchased from Aquaculture Technical Developing Company, Hebei Province, China. The chemical structures of LA, bupivacaine, and tetracaine are shown in Fig. 1.

cDNA and mutagenesis

The plasmid with the human Nav1.7 CDNA insert was kindly donated by Dr. Norbert Klugbauer (Albert-Ludwigs-Universität Freiburg, Freiburg, Germany). The human Nav1.3, Nav1.4, Nav1.5, and Nav1.8 cDNAs were synthesized and inserted into the proper sites of the pcDNA3.1 (+) vector by Sangon Biotech, Shanghai. Point mutations in Nav1.7 were individually constructed by sitedirected mutagenesis and confirmed by sequencing.

Cell culture and transfection

The wild-type human Nav1.7 channel was stably expressed in human embryonic kidney (HEK293) cells, and the cell lines were grown in high-glucose DMEM (Gibco, Carlsbad, CA, USA) supplemented with $10 \%$ fetal bovine serum and selected with $300 \mu \mathrm{g} / \mathrm{mL}$ antibiotic hygromycin B (Invitrogen, Carlsbad, CA, USA) at $37^{\circ} \mathrm{C}$ in a humidified atmosphere with $5 \% \mathrm{CO}_{2}$. Chinese hamster ovary $(\mathrm{CHO})$ cells were used to express the sodium isoforms (Nav1.3, Nav1.4, and Nav1.5) and Nav1.7 mutants. The CHO cells were cultured in 50/50 DMEM/F-12 (Gibco, Carlsbad, CA, USA) supplemented with $10 \%$ fetal bovine serum. Two days prior to recording, the corresponding sodium channel plasmids were transfected into $\mathrm{CHO}$ cells with Lipofectamine reagent (Invitrogen, Carlsbad, CA, USA). The hNav1.8 plasmids were transfected in the dorsal root ganglion-neuroblastoma ND7/23 cell line, which was a generous gift from Lan Bao's laboratory (Institute of Biochemistry and Cell Biology, Chinese Academy of Sciences, Shanghai, China) and was cultured in high-glucose DMEM supplemented with $10 \%$ fetal bovine serum. To aid the identification of transfected cells by fluorescence microscopy, a GFP construct was co-transfected with all Nav1.7 mutants and Nav1.3, Nav1.4, Nav1.5, and Nav1.8. Before electrophysiological recordings, all cells were plated onto poly- $L$ lysine-coated glass coverslips and used within $24 \mathrm{~h}$.

\section{Electrophysiology}

Whole-cell patch-clamp recordings of activated sodium channel membrane currents were acquired at room temperature using an Axopatch 700B amplifier (Axon Instruments, Burlingame, CA, USA). Pipettes were pulled from borosilicate glass capillaries (World Precision Instruments, Sarasota, FL, USA) with an open-tip resistance typically ranging from 1.5 to $3 \mathrm{M} \Omega$ when filled with intracellular solution. The recording pipette intracellular solution contained the following (in $\mathrm{mmol} / \mathrm{L}$ ): $140 \mathrm{CsF}, 10 \mathrm{NaCl}, 10 \mathrm{HEPES}$, 1.1 EGTA, and 20 glucose (pH 7.3 adjusted by $\mathrm{CsOH}$ ); the bath or extracellular solution contained the following (in $\mathrm{mmol} / \mathrm{L}$ ): 140 $\mathrm{NaCl}, 3 \mathrm{KCl}, 1 \mathrm{MgCl}_{2}, 1 \mathrm{CaCl}_{2}, 10$ HEPES, and 20 glucose $(\mathrm{pH} 7.3$ adjusted by $\mathrm{NaOH}$ ). When the perfusion method was used, the bath solution was continuously perfused using a BPS perfusion system (ALA Scientific Instruments, Westburg, NY, USA). Recording was performed after a 4-min equilibration period at $-80 \mathrm{mV}$ after the whole-cell configuration was achieved. When the incubation method was used, cells were incubated with LA or extracellular solution (added in the same volume of DMSO) for $20 \mathrm{~min}$ before they were patched, and then recording was performed after a 4min equilibration period at $-80 \mathrm{mV}$ after the whole-cell configuration was achieved. The perfusion method was used for all electrophysiological experiments except when mentioned in this paper. Series resistance compensation was used and set to $80 \%$. 

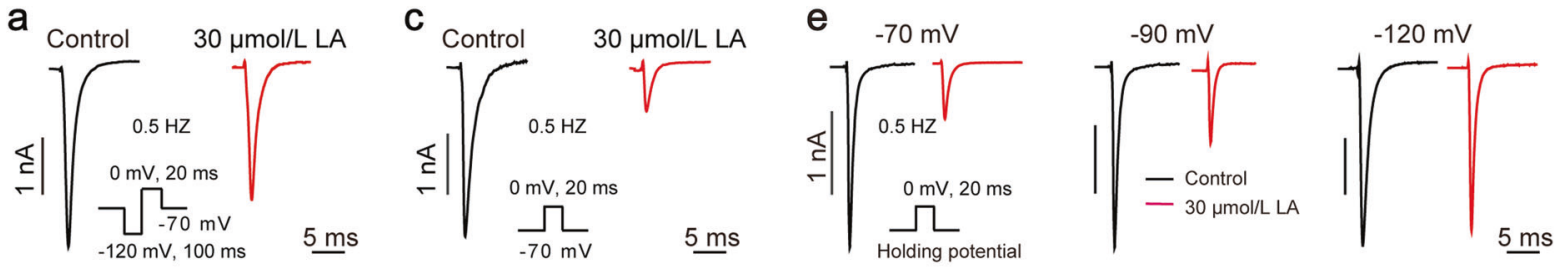

b

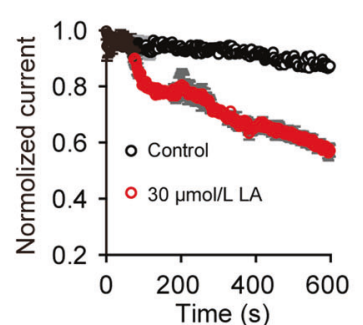

d

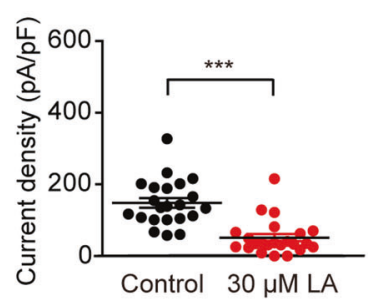

f

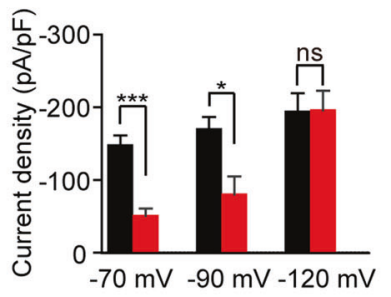

g

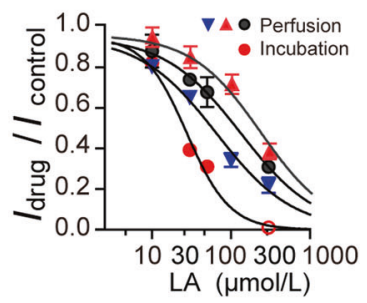

Fig. 2 Characterization of LA inhibition of the Nav1.7 channel. a Representative Nav1.7 currents before and at the time point 10 min after bath perfusion of $30 \mu \mathrm{mol} / \mathrm{L}$ LA. (Inset) The experimental protocol. The currents were elicited by a $20-\mathrm{ms}$ test pulse to $0 \mathrm{mV}$ at $0.5 \mathrm{~Hz}$ from a holding potential of $-70 \mathrm{mV}$, at which half of the hNav1.7 channels would move into the inactivation state. To relieve inactivation during the repetitive stimulus, a short 100-ms pulse to $-120 \mathrm{mV}$ was applied before the test pulse. b Time courses of hNav1.7 currents with or without bath perfusion of $30 \mu \mathrm{mol} / \mathrm{L} \mathrm{LA}$ ( $n=3 \mathrm{for}$ control and $30 \mu \mathrm{mol} / \mathrm{L} \mathrm{LA})$. c Representative hNav1.7 currents elicited from cells after incubation with or without $30 \mu \mathrm{mol} / \mathrm{L}$ LA for $20 \mathrm{~min}$. d Scatter plot showing the current densities of hNav1.7 channels incubated with or without $30 \mu \mathrm{mol} / \mathrm{L}$ LA for 20 min. e Representative hNav1.7 currents elicited from cells at a holding potential of $-70,-90$, and $-120 \mathrm{mV}$ after incubation with or without $30 \mathrm{\mu mol} / \mathrm{L}$ for $20 \mathrm{~min}$. f Bar graph summarizing the current densities of hNav1.7 channels calculated from e $(-70 \mathrm{mV}: n=22$ for control, $n=23$ for $30 \mu \mathrm{mol} / \mathrm{L} \mathrm{LA} ;-90 \mathrm{mV}: n=4$ for control, $n=5$ for $30 \mu \mathrm{mol} / \mathrm{L}$ LA; $-120 \mathrm{mV}: n=11$ for control, $n=10$ for $30 \mu \mathrm{mol} / \mathrm{L} \mathrm{LA})$. g The dose-response curves of LA for hNav1.7 channels under the indicated recording conditions. Concentration-dependent inhibition of hNav1.7 at the holding potential of $-50 \mathrm{mV}$ (blue triangles), $-70 \mathrm{mV}$ (black circles), and $-120 \mathrm{mV}$ (red triangles) after perfusion of LA for 8-9 min. The fitted $\mathrm{IC}_{50}$ values were $65.33(51.94-78.71) \mu \mathrm{mol} / \mathrm{L}(-50 \mathrm{mV}), 133.20(92.91-173.40) \mu \mathrm{mol} / \mathrm{L}(-70 \mathrm{mV})$, and $221.30(168.80-273.80) \mu \mathrm{mol} / \mathrm{L}$ $(-120 \mathrm{mV})$ under the perfusion condition. When the $\mathrm{IC}_{50}$ value was measured at a holding potential of $-70 \mathrm{mV}$ using the incubation method, the $\mathrm{IC}_{50}$ value decreased to approximately $27.67(15.68-39.66) \mu \mathrm{mol} / \mathrm{L}\left(n \geq 3\right.$ for each condition, $\left.{ }^{* * *} P<0.001,{ }^{*} P<0.05\right)$

Data and statistical analysis

The data and statistical analysis complied with the recommendations on experimental design and analysis in pharmacology. Patch-clamp data were processed using Clampfit 10.4 (Molecular Device, Sunnyvale, CA, USA) and analyzed with GraphPad Prism 5.0 (GraphPad Software, San Diego, CA, USA). Peak inward currents obtained from activation protocols were converted to conductance values using the equation: $G=I /\left(V-V_{\mathrm{Na}}\right) . V_{\mathrm{Na}}$ indicates the reversal potential of sodium currents. To measure inactivation, the peak currents after different prepulses were normalized and plotted as a function of voltage. To quantify these biophysical properties, we fitted the activation and steady-state inactivation curves with the Boltzmann equation: $Y=$ Bottom + (Top-Bottom)/ $\left(1+\exp \left[\left(V_{1 / 2}-V_{m}\right) / k\right]\right), Y$ is $G / G_{\max }$ or $I / I_{\max }, V_{1 / 2}$ is the voltage at which $50 \%$ of the maximum conductance (or current) was obtained, and $k$ is the slope factor. Dose-response curves were fitted with a 3-parameter Hill equation: $Y=$ Bottom + $($ Top-Bottom $) /\left(1+10^{\wedge}(X\right.$-LoglC 50$\left.)\right)$, where Bottom and Top are the minimum and maximum inhibition, respectively, $X$ is the concentration of LA, $Y$ is the value of $I_{\text {drug }} / I_{\text {control, }}$ and $I C_{50}$ is the drug concentration producing a half-maximum response. Due to the low solubility of LA, we were unable to test concentrations higher than $300 \mu \mathrm{mol} / \mathrm{L}$. In addition, we attempted to increase the concentration of LA up to $100 \mu \mathrm{mol} / \mathrm{L}$ by applying the incubation method but found that the cells could not be patched. Therefore, the highest concentration used when applying the incubation method was $50 \mu \mathrm{mol} / \mathrm{L}$, which is depicted as the hollow red dot in the dose-response curves reflecting the 3-parameter Hill equation: $Y=$ Bottom $+($ Top-Bottom $) /\left(1+10^{\wedge}\left(X\right.\right.$-LoglC $\left.\left.{ }_{50}\right)\right)$. The results are presented as the mean \pm SEM. The significance was estimated using unpaired two-tailed $t$-tests, and a $P<0.05$ was considered statistically significant. Detailed information is provided in the figure legends.

\section{RESULTS}

LA exerts a voltage-dependent inhibition of the Nav1.7 channel The inhibitory activity of LA on the hNav1.7 channel was examined using the whole-cell voltage-clamp technique. To elicit Nav1.7 currents, a 20-ms test pulse to $0 \mathrm{mV}$ from a holding potential of $-70 \mathrm{mV}$ was applied. We found that the peak currents slowly decreased after bath perfusion of $30 \mu \mathrm{mol} / \mathrm{L}$ LA at the holding potential of $-70 \mathrm{mV}$, at which approximately half of the Nav1.7 channels were inactivated. However, the inhibitory potency of LA was unable to reach steady-state even 8-9 min after bath perfusion of the drug (Fig. 2a, b). To investigate the LA effect in a longer duration and minimize the current rundown after membrane rupture, we used the incubation method. The cells were incubated in solutions with or without LA for $20 \mathrm{~min}$. Then, the currents of Nav1.7 channels were recorded using whole-cell patch clamp. We observed a stronger inhibition after an extended incubation duration. The relative Nav1.7 current density dramatically decreased by approximately $66 \%$ (control: $-147.69 \pm 13.68$ $\mathrm{pA} / \mathrm{pF}(n=22)$ vs. LA: $-50.84 \pm 10.14 \mathrm{pA} / \mathrm{pF}(n=23), P<0.01)$ after incubation with $30 \mu \mathrm{mol} / \mathrm{L}$ LA for $20 \mathrm{~min}$ at the same holding potential $(-70 \mathrm{mV})$ as that applied in the perfusion method (Fig. 2c, d). When the holding potential was changed to $-90 \mathrm{mV}$, which would inactivate approximately $10-20 \%$ of Nav1.7 channels, the reduction in the current density by $30 \mu \mathrm{mol} / \mathrm{L}$ LA decreased to approximately 47\% (control: $-169.96 \pm 16.53 \mathrm{pA} /$ $\mathrm{pF}(n=4)$ vs. LA: $-80.34 \pm 24.57 \mathrm{pA} / \mathrm{pF}(n=5), P<0.05)$. Notably, when the cells were clamped at $-120 \mathrm{mV}$, a membrane potential that would prevent channel inactivation, the inhibitory effect of LA on Nav1.7 channels disappeared (control: $-193.97 \pm 25.44$ $\mathrm{pA} / \mathrm{pF}(n=11)$ vs. LA: $-195.72 \pm 27.19 \mathrm{pA} / \mathrm{pF}(n=10), P=0.9629)$ (Fig. 2e, f). The dose-response curves of LA were also measured using incubation or perfusion methods. The dose-dependent inhibition of LA using the perfusion method was measured 

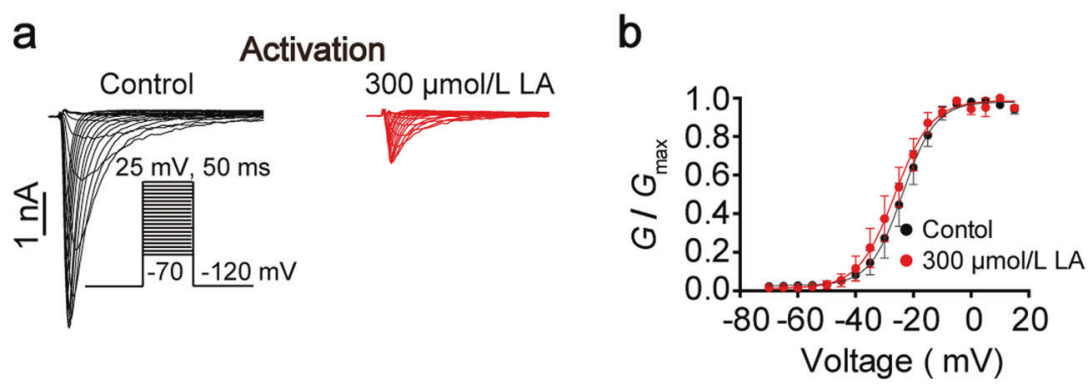

C
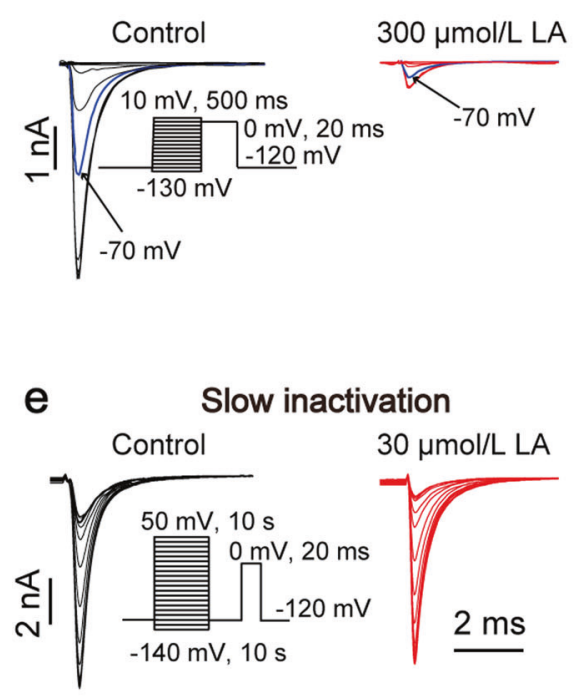

d

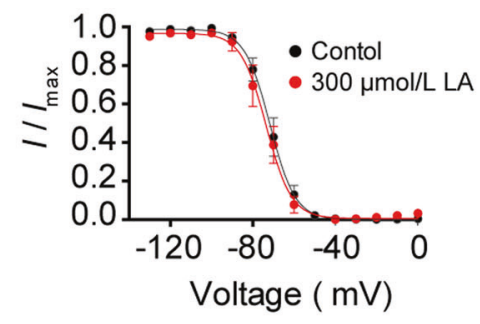

f

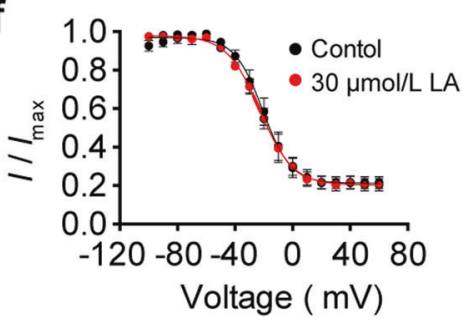

Fig. 3 LA does not affect the voltage dependence of the hNav1.7 channel. a Representative activation current traces of Nav1.7 with or without $300 \mu \mathrm{mol} / \mathrm{L}$ LA. (Inset) The recording protocol. The currents were elicited using a range of depolarizing potentials $(-70 \mathrm{mV}$ to $+25 \mathrm{mV}$ ) from a holding potential of $-120 \mathrm{mV}$ at a stimulus frequency of $0.5 \mathrm{~Hz}$. b Activation curves of Nav1.7 channels with or without $300 \mu \mathrm{mol} / \mathrm{L} \mathrm{LA}$ measured from a $(n=5)$. c Representative fast inactivation current traces of Nav1.7 with or without $300 \mu \mathrm{mol} / \mathrm{L}$ LA. The steady-state fast inactivation was evaluated by a test pulse stepped to $10 \mathrm{mV}$ after $500-\mathrm{ms}$ prepulses to varying voltage potentials from a holding of $-120 \mathrm{mV}$ at a stimulus frequency of $0.5 \mathrm{~Hz}$. d The voltage dependence of the steady-state fast inactivation curves with or without $300 \mu \mathrm{mol} / \mathrm{L} \mathrm{LA}$ measured from c $(n=6)$. e Representative slow inactivation current traces of Nav1.7 after incubation with or without $30 \mu \mathrm{mol} / \mathrm{L}$ LA for $20 \mathrm{~min}$. The steady-state slow inactivation was examined using 10-s depolarization prepulses followed by a 20-ms hyperpolarization pulse to - $120 \mathrm{mV}$ to allow channels to recover from fast inactivation, and a test pulse to $0 \mathrm{mV}$ for $20 \mathrm{~ms}$ was then applied to determine the channel availability for $13 \mathrm{~s} /$ trace. $\mathbf{f}$ The voltage dependence of the steady-state slow inactivation curve with or without $30 \mu \mathrm{mol} / \mathrm{L} \mathrm{LA}$ measured from e $(n=4)$

under three holding potentials. The $\mathrm{IC}_{50}$ values were 65.33 (51.94-78.71), 133.20 (92.91-173.40), and 221.30 (168.80-273.80) $\mu \mathrm{mol} / \mathrm{L}$ when the holding potentials were clamped at -50 , -70 , and $-120 \mathrm{mV}$, respectively (Fig. $2 \mathrm{~g}$ ). When the doseresponse curve of $L A$ was measured using the incubation method, the $\mathrm{IC}_{50}$ value at a holding potential of $-70 \mathrm{mV}$ decreased to 27.67 (15.68-39.66) $\mu \mathrm{mol} / \mathrm{L} \quad(n \geq 4)$ (Fig. 2g). Collectively, these data showed that LA is a voltage-dependent Nav1.7 inhibitor.

LA does not affect activation or inactivation of the hNaV1.7 channel

To understand whether LA modifies the activation of the hNav1.7 channel, we stepped the cells to various potentials between -70 and $+25 \mathrm{mV}$ in $5-\mathrm{mV}$ increments for $50 \mathrm{~ms}$ from a holding potential of $-120 \mathrm{mV}$. Representative current traces before and after bath perfusion with $300 \mu \mathrm{mol} / \mathrm{L}$ LA are shown in Fig. 3a. The $V_{1 / 2}$ values before and after perfusion of LA were $-23.71 \pm 0.78 \mathrm{mV}$ and $-26.95 \pm 1.02 \mathrm{mV}(n=5, P>0.05)$, respectively (Fig. 3b). Next, the impact of LA on Nav1.7 channel inactivation was investigated. Nav1.7 channel inactivation can be divided into two separate states, i.e., fast and slow inactivation [29]. The steady-state fast inactivation of Nav1.7 was determined using a 500-ms conditioning pulse from -130 to $+10 \mathrm{mV}$ in $10-\mathrm{mV}$ increments, followed by a $0-\mathrm{mV}$ test pulse. Representative traces generated with or without $300 \mu \mathrm{mol} / \mathrm{L}$ LA are shown in Fig. 3c. The $V_{1 / 2}$ values before and after bath perfusion of LA were $-71.70 \pm 0.75 \mathrm{mV}$ and $-73.51 \pm 1.05 \mathrm{mV}(n$ $=6, P>0.05)$, respectively (Fig. $3 \mathrm{~d}$ ). The steady-state slow inactivation was measured using 10-s depolarization prepulses followed by a $20-\mathrm{ms}$ hyperpolarization pulse to $-120 \mathrm{mV}$ to allow channels to recover from the fast inactivation before the test pulse to determine channel availability. Providing that a recovery interval of $3 \mathrm{~s}$ at $-120 \mathrm{mV}$ is sufficient to re-prime Nav1.7 after the 10-s prepulses, a fixed 13-s pulse duration was applied during the slow inactivation study. To minimize rundown during the long-duration recording, the effect of LA on slow inactivation was evaluated using the incubation method. After incubation with $30 \mu \mathrm{mol} / \mathrm{L}$ LA for $20 \mathrm{~min}$, the steady-state slow inactivation of Nav1.7 was similar to that in the control (Fig. 3e). The $V_{1 / 2}$ values with and without LA were $-20.76 \pm 1.42 \mathrm{mV}$ and $-22.73 \pm 1.39 \mathrm{mV}(n=4$ for each group, $P$ $>0.05$ ), respectively (Fig. $3 \mathrm{f}$ ). We speculated that the lack of effects of LA on Nav1.7 channel slow inactivation might result from lack of inhibition on the resting state of the channels, as shown in Fig. 2f. Taken together, our data indicated that LA does not affect the activation or inactivation of the Nav1.7 channel. 
a
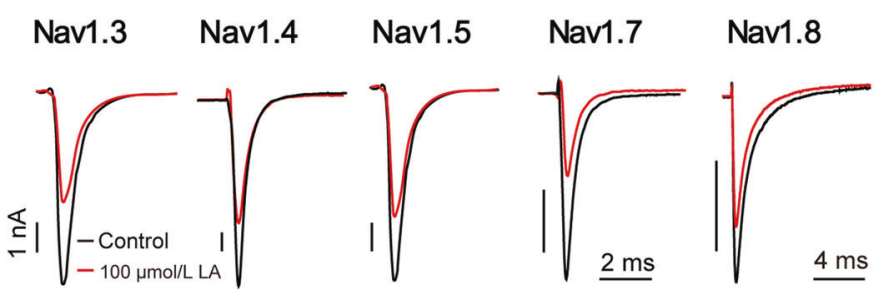

C
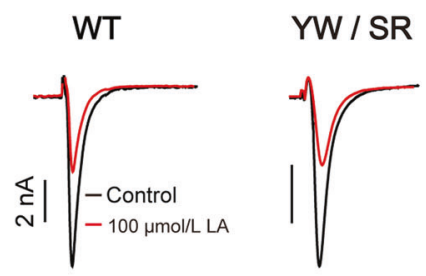

e

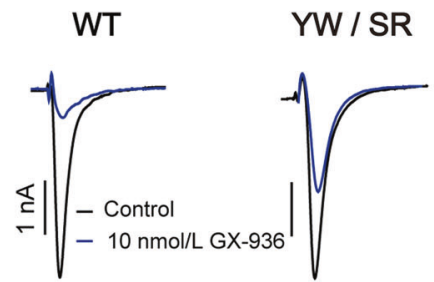

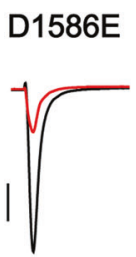

D1586E

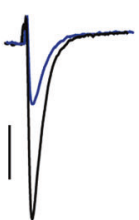

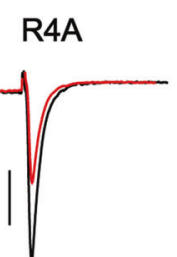

R4A

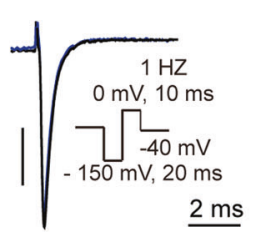

b
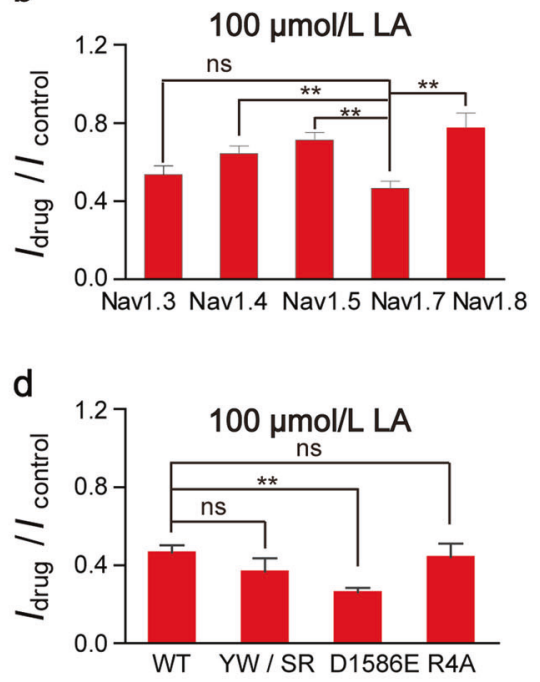

f

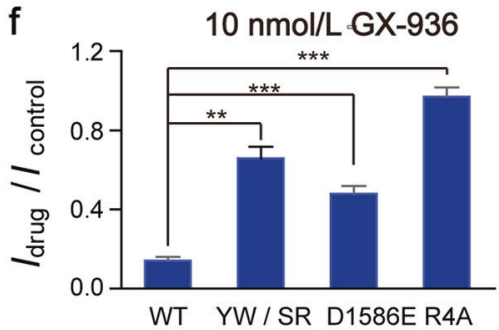

Fig. 4 Inhibitory activities of LA on other Nav channels. a Representative traces of a panel of human Nav channels before (black) and after (red) bath perfusion of $100 \mu \mathrm{mol} / \mathrm{L}$ LA. b Bar graph showing the potency of $100 \mu \mathrm{mol} / \mathrm{L}$ LA on different human Nav isoforms measured from a $(n=4$ for Nav1.3, $n=5$ for Nav1.4, $n=3$ for Nav1.5, $n=7$ for Nav1.7, and $n=3$ for Nav1.8). c Representative current traces of a panel of Nav1.7 mutants YW/SR (Y1537S/W1538R), D1586E, and R4 (R1608A) before (black) and after (red) bath perfusion of 100 $\mu \mathrm{mol} / \mathrm{L}$ LA. The experiments $(\mathbf{a}, \mathbf{c})$ were recorded using the identical protocol as in Fig. 2a. d Histogram shows the inhibitory effect of $100 \mu \mathrm{mol} / \mathrm{L}$ LA on the mutants as indicated ( $n=7$ for WT, $n=3$ for YW/SR, $n=4$ for D1586E, and $n=3$ for R1608A). e Representative current traces of Nav1.7 mutants before (black) and after (blue) perfusion of $10 \mathrm{nmol} / \mathrm{L} \mathrm{GX}-936$. To activate Nav1.7 currents, a short 20-ms prepulse to -150 mV was used to recover channels from inactivation, followed by a $10-\mathrm{ms}$ pulse to $0 \mathrm{mV}$ to open the channels at a 1- $\mathrm{Hz}$ pulse rate. The holding potential was maintained at $-40 \mathrm{mV}$. The experimental protocol applied was consistent to those in previous reports. $f$ Histogram shows a significant reduction in the inhibitory efficacy of GX-936 on the mutants measured from e. ${ }^{* *} P<0.001,{ }^{* *} P<0.01$

LA inhibits other peripheral Nav isoforms

In addition to Nav1.7, some other Nav isoforms distributed in the peripheral tissues also contribute to the physiological or pathophysiological electrical signaling in excitable cells [30]. Therefore, the effects of LA on a portion of these isoforms were further evaluated. We found that perfusion with $100 \mu \mathrm{mol} / \mathrm{L} \mathrm{LA}$ inhibited the currents of Nav1.3 $(46 \% \pm 4 \%, n=4)$, Nav1.4 $(38 \% \pm$ $4 \%, n=5)$, Nav1.5 (29\% $\pm 4 \%, n=3)$, and Nav1.8 $(22 \% \pm 7 \%, n=3)$ (Fig. $4 \mathrm{a}, \mathrm{b}$ ). Compared to the inhibition of these isoforms, the inhibitory efficacy of LA on the Nav1.7 channel was more potent at $100 \mu \mathrm{mol} / \mathrm{L}(53 \% \pm 4 \%, n=7)$. In the Nav channels, there are at least six different binding sites located either at the pore region or the VSD known for binding toxins and synthetic derivatives, classified as neurotoxin receptor site 1-6 [31]. Residues from the S2 and S3 helices of the fourth VSD (DIV) have been demonstrated to be the binding sites for aryl sulfonamide antagonists such as GX-936, a highly selective Nav1.7 channel inhibitor [32]. Mutations of these residues that are responsible for Nav1.7 selectivity such as Y1537R/W1538S, D1586E, and R4A significantly decreased the GX936-induced inhibition of the channel (Fig. 4e, f). In contrast, all of these mutations failed to impair the inhibitory activity of LA on Nav1.7, which is consistent to the lack of voltage dependence of LA, as these mutants are located at the S2 and S3 helices of the fourth VSD (Fig. 4c, d).
The local anesthetic site is important to LA tonic inhibition The unsatisfactory Nav channel subtype selectivity suggested that LA may behave similar to local anesthetics that non-selectively inhibit Nav channels and bind to the intracellular pore region [33]. The inhibitory activities of local anesthetics, such as tetracaine and bupivacaine, on the Nav1.5 channel have been well characterized, and multiple residues that are located in the intracellular portion of the channel have been identified as essential for the inhibitory effects of local anesthetics [33]. These essential residues are highly conserved between hNav1.5 and hNav1.7 channels (Fig. 5a). The corresponding residues in Nav1.7 were mutated to either alanine (A) or lysine $(K)$, and the responses of these mutants to $L A$, tetracaine, and bupivacaine were individually evaluated using the bath-perfusion method. At the end of each recording, $1 \mu \mathrm{mol} / \mathrm{L}$ tetrodotoxin (TTX) was perfused to verify that the currents were recorded from TTX-sensitive Nav1.7. We found that the local anesthetic site N395 in DI was not necessary for the effects of any of the three tested drugs. The local anesthetic sites in both DIII (L1438 and/or I1442) and DIV (F1737, N1742, and Y1744) were required for the effects of tetracaine and bupivacaine. However, the residues in DIII were not important for the inhibitory activity of LA. In addition, the residue Y1744 in DIV was also not required (Fig. 5b-e). These data revealed that the residues at the local anesthetic site in DIV are important for the inhibitory activity of LA. 
a

Domain I

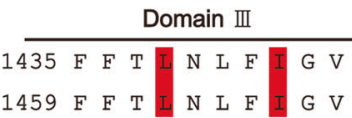

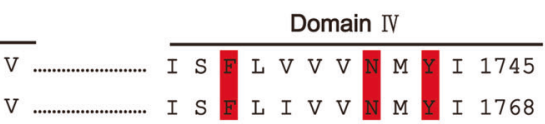

b hNav1.7 388 L G S F Y L I N I
hNav1.5 399 L G S F Y L L V I $\ldots \ldots \ldots \ldots \ldots$ L1438A I1442A F1737K N1742K Y1744K $100 \mu \mathrm{M}$ LA
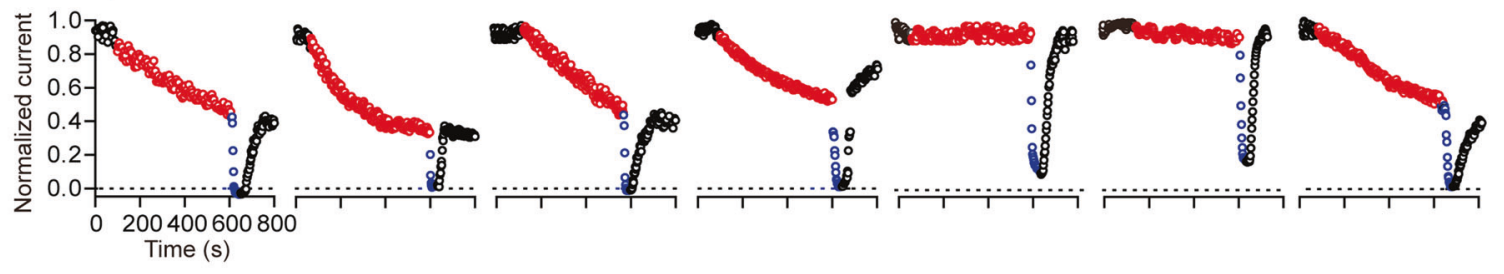

\section{$2 \mu \mathrm{M}$ tetracaine}
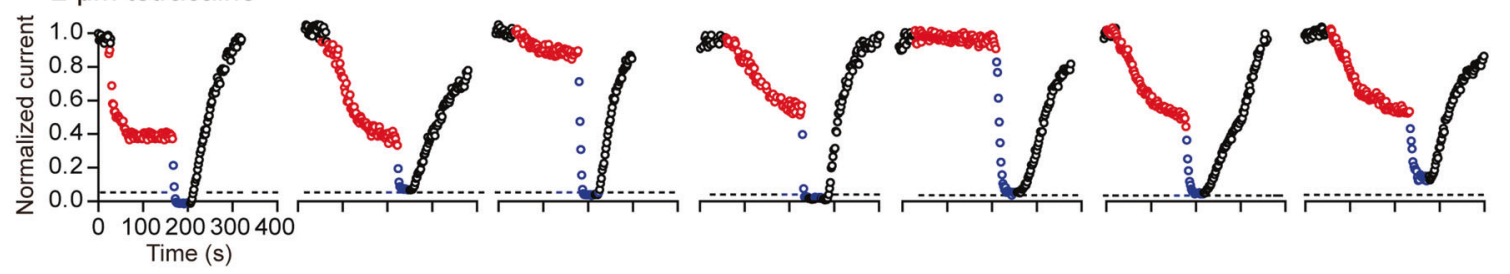

$20 \mu \mathrm{M}$ bupivacaine
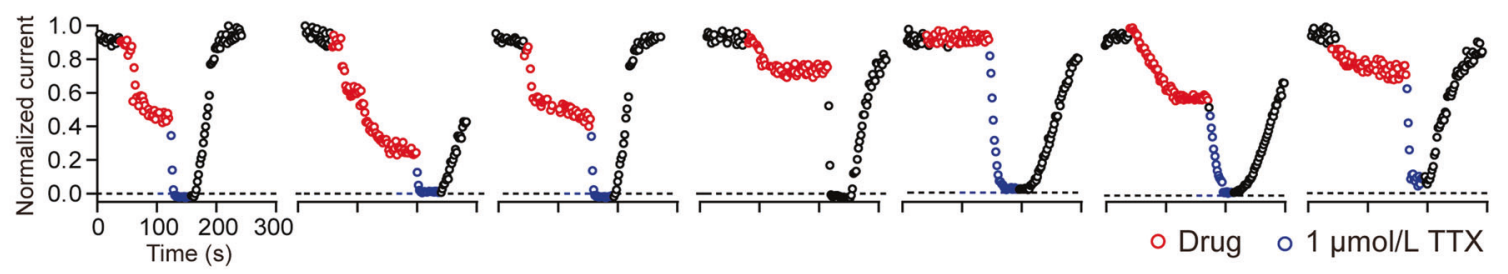

C

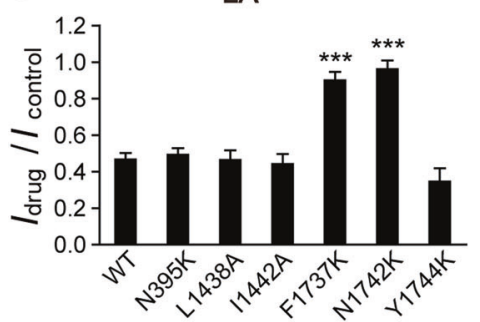

d

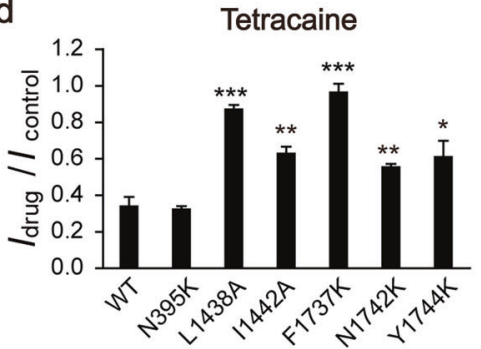

e

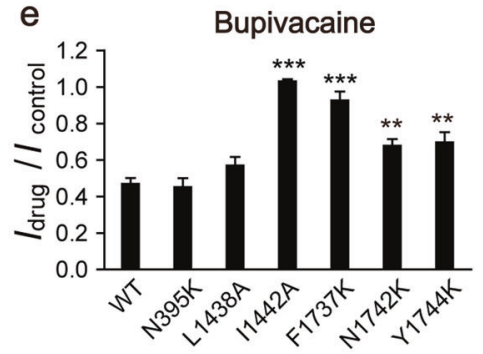

Fig. 5 Molecular determinants of LA inhibitory activity. a Alignment of intracellular residues on S6 of Nav1.5 and Nav1.7. The residues responsible for the binding of local anesthetics are highlighted in red. b Representative time courses of WT or Nav1.7 mutants with (red cycles) and without (black cycles) $100 \mu \mathrm{mol} / \mathrm{L} \mathrm{LA}, 20 \mu \mathrm{mol} / \mathrm{L}$ bupivacaine, or $2 \mu \mathrm{mol} / \mathrm{L}$ tetracaine. To verify the currents, $1 \mu \mathrm{mol} / \mathrm{L}$ TTX (blue cycles) was applied to all cells and then washed out with control solution. Histograms show the inhibitory effect of LA (c), tetracaine (d), and bupivacaine (e) on WT and mutants measured from $\mathbf{b}\left(n \geq 3,{ }^{* *} P<0.001,{ }^{*} P<0.01, * P<0.05\right)$

LA inhibits the Nav1.7 channel in a different manner from common local anesthetics

Although the mutant experiments revealed that LA may bind to different sites from local anesthetics tetracaine and bupivacaine, the difference seemed insufficient to explain the featured analgesic activities of LA such as the long maintenance time [7, 8]. In the initial experiments, we showed that the inhibition of Nav1.7 by $30 \mu \mathrm{mol} / \mathrm{L}$ LA developed very slowly and was unable to reach steady-state $8-9 \mathrm{~min}$ after perfusion of the drug (Fig. 2b). The reversibility of the slow inhibition was further examined under a higher concentration $(300 \mu \mathrm{mol} / \mathrm{L})$ of LA. Approximately $8 \mathrm{~min}$ after perfusion of $L A$, the inhibition reached steady state, and approximately $80 \%$ of the current was suppressed. However, removal of LA by switching to bath solutions without LA was unable to restore the Nav1.7 currents. Furthermore, current recovery was not observed after an extended washout duration of $20 \mathrm{~min}$. In contrast, for tetracaine and bupivacaine, the inhibitory effect developed relatively fast, and the inhibited currents were completely restored in a couple of minutes after removal of the drugs (Fig. 6b-e).

To date, a number of Nav1.7 inhibitors with different effects on channel activation and inactivation have been developed, such as CNV1014802, PF-05089771, and XEN402 [27]. The influence of one inhibitor on channel activation or inactivation may affect its analgesic activity [34]. Notably, LA exhibited a different pattern from tetracaine and bupivacaine in terms of the influence on channel activation and inactivation (Fig. 6f). Neither activation nor inactivation were changed after perfusion of $300 \mu \mathrm{mol} / \mathrm{L} \mathrm{LA}$. Bath perfusion of $2 \mu \mathrm{mol} / \mathrm{L}$ tetracaine dramatically right-shifted the $V_{1 / 2}$ of the voltage-dependent activation by approximately $8.92 \pm 0.80$ $\mathrm{mV}$ (control: $-19.34 \pm 0.56 \mathrm{mV}$ vs. tetracaine: $-10.42 \pm 1.03 \mathrm{mV}$, $n=3, P<0.01)$, whereas tetracaine had no effects on channel 
a

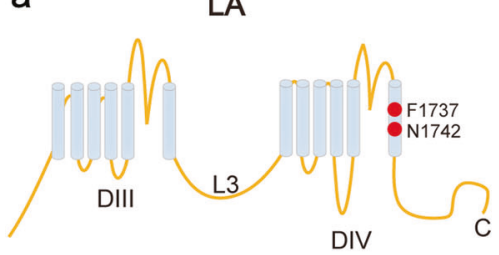

b

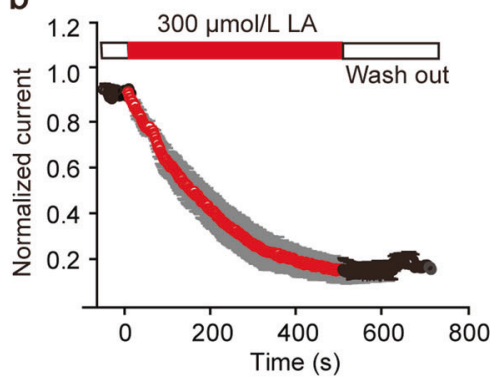

Tetracaine

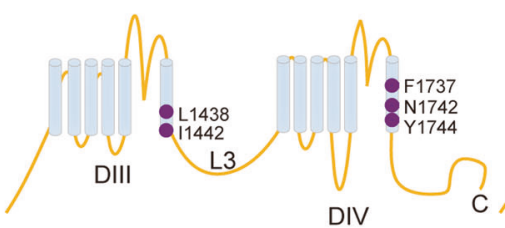

C
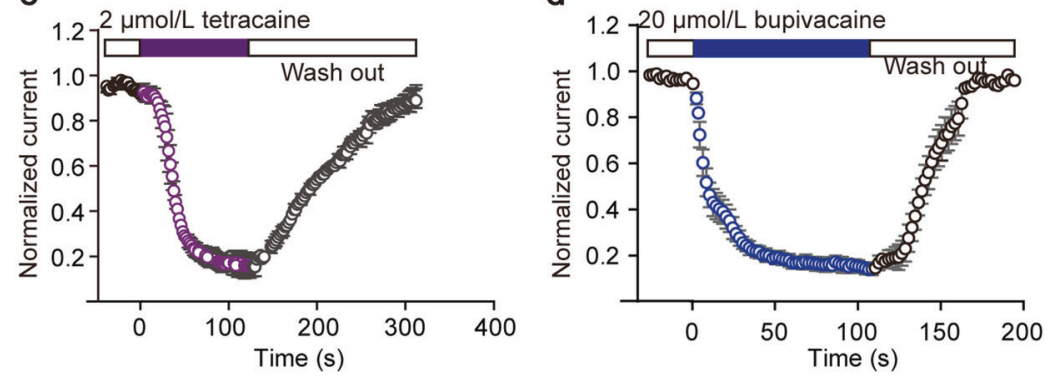

e

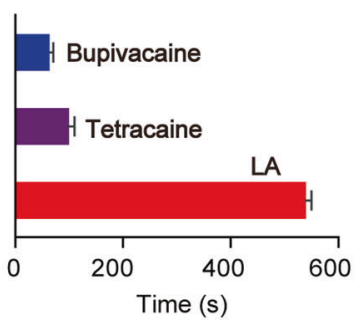

f

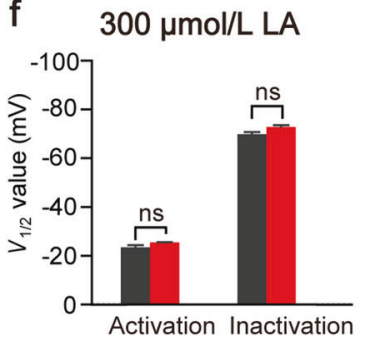

$2 \mu \mathrm{mol} / \mathrm{L}$ tetracaine

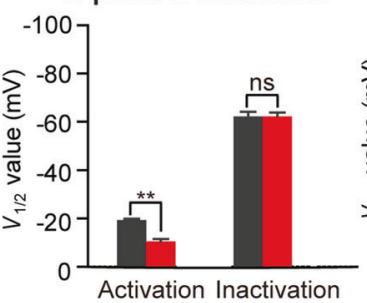

d

Bupivacaine

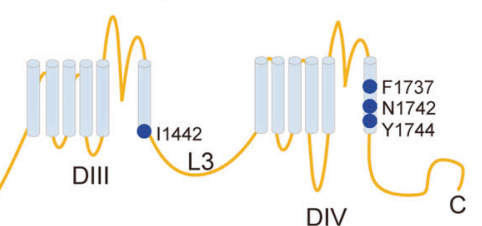

DIV

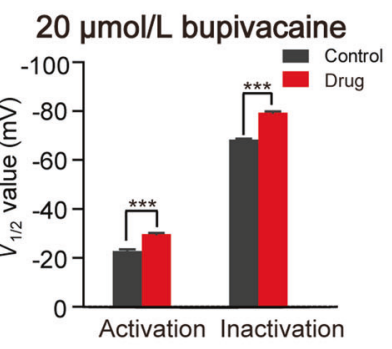

Fig. 6 Comparison of the inhibitory effects of LA, bupivacaine, and tetracaine on Nav1.7. a Cartoons show the key residues that are responsible for the LA, bupivacaine, and tetracaine inhibition of Nav1.7. b, c Representative time courses of Nav1.7 channel currents in the absence and presence of $300 \mu \mathrm{mol} / \mathrm{L} \mathrm{LA}(\mathbf{b}), 2 \mu \mathrm{mol} / \mathrm{L}$ tetracaine (c), and $20 \mu \mathrm{mol} / \mathrm{L}$ bupivacaine $(\mathbf{d})(n=3)$. The experimental protocol was the same as that in Fig. 2a. e Bar graph shows the duration to reach steady state with the indicated drugs measured from the data in b to d. f Histograms show the $V_{1 / 2}$ values of the activation and fast inactivation in the absence (black) and presence of drugs (red) as indicated ( $n=6$ for $2 \mu \mathrm{mol} / \mathrm{L}$ tetracaine, $n=4$ for $20 \mu \mathrm{mol} / \mathrm{L}$ bupivacaine, ${ }^{* * *} P<0.001,{ }^{* *} P<0.01$ )

inactivation (control: $-62.20 \pm 1.94 \mathrm{mV}$ vs. tetracaine: $-62.18 \pm$ $1.64 \mathrm{mV}, n=6, P>0.05$ ). Bupivacaine affected both activation and inactivation. After perfusion of $20 \mu \mathrm{mol} / \mathrm{L}$ bupivacaine, the $V_{1 / 2}$ of the voltage-dependent activation was left-shifted by approximately $6.83 \pm 0.48 \mathrm{mV}$ (control: $-22.86 \pm 0.52 \mathrm{mV}$ vs. bupivacaine: $-29.69 \pm 0.44 \mathrm{mV}, n=4, P<0.001$ ), and the $V_{1 / 2}$ of the voltagedependent inactivation was left-shifted by approximately $11.06 \pm$ $0.44 \mathrm{mV}$ (control: $-68.34 \pm 0.38 \mathrm{mV}$ vs. bupivacaine: $-79.40 \pm 0.50$ $\mathrm{mV}, n=4, P<0.001)$. Considering the slow onset, irreversibility, and lack of influence on channel activation and inactivation accompanied with the different molecular determinants, we proposed that LA may inhibit Nav1.7 in a manner different from local anesthetics.

\section{DISCUSSION}

Although several subtypes of Nav channels have been suggested to play a role in pain signaling, Nav1.7 has received immense attention due to the remarkable human phenotypes caused by its inherited mutations $[25,26]$. Loss-of-function mutations in Nav1.7 cause congenital insensitivity to pain, resulting in an inability to feel pain with few sensory impairments [35]. This feature of Nav1.7 is dramatically different from those of previous reported painless targets, such as neurotrophic tyrosine kinase (Trk-A) and nerve growth factor $\beta$ (NGF $\beta$ ), mutations of which may damage intelligence or autonomic functions [36]. Because Nav1.7 is a human-validated analgesic target, a number of Nav1.7 channel inhibitors, including antibodies, toxins, and small molecules, have been developed [27, 34]. In 2013, one small molecule named XEN402 or TV-45070 was granted an orphan drug designation by the US Food and Drug Administration for the treatment of pain associated with erythromelalgia [37].

In the current study, we found that $10 \mu \mathrm{mol} / \mathrm{L}$ LA could produce an obvious inhibition on Nav1.7 channel (Fig. 2g). We further provided evidence that LA is an inhibitor of the Nav1.7 channel with an $\mathrm{IC}_{50}$ value of $27.67(15.68-39.66) \mu \mathrm{mol} / \mathrm{L}$ at a holding potential of $-70 \mathrm{mV}$ (Fig. $2 \mathrm{~g}$ ). According to the study conducted by Ameri and his colleagues, the effective concentration of LA in mouse plasma would be in the range of $5-9 \mu \mathrm{mol} / \mathrm{L}$ [18]. Previous studies have shown that LA does not inhibit voltage-dependent potassium currents, calcium currents, or ligands, such as glutamate, GABA, glycine, and ATP, -induced currents in isolated trigeminal ganglia neurons [20]. The subtype selectivity study showed that LA exhibits a more potent inhibition of Nav1.7 than that on other isoforms that are also expressed in the peripheral system (Fig. 4b). The inhibitory effect on Nav1.3 and Nav1.8 may also contribute to the analgesic activity of LA since these two isoforms also participate in pain signaling, though the inhibitory potency on these two isoforms is slightly weaker than that on Nav1.7 [38]. Nav1.4 is majorly expressed in skeletal muscle, and mutations in Nav1.4 cause muscle stiffness or weakness, two opposite clinical symptoms [39]. Nav1.5 is predominantly expressed in cardiac tissue. The observed inhibitory effect on Nav1.5 is consistent to the previous reports that LA can cause arrhythmia in the guinea pig heart [40]. Taken together, these data suggested that the inhibition of Nav channels, particularly Nav1.7, 
might be one of the mechanisms underlying the analgesic activity of LA, especially for its local application. For further determination of the roles of Nav1.7 or other pain-related isoforms, in vivo experiments using transgenic mice or a scorpion toxin OD-1, a Nav1.7-specific agonist, -induced pain model will be helpful [41].

The inhibitory potency of LA on the Nav1.7 channel increased as the holding potential was depolarized (Fig. 2e). These data indicated that LA inhibits the Nav1.7 channel in a voltagedependent manner. In the current study, we found that the inhibitory efficacy of LA is closely correlated with the channel open time but not the stimulation rate (Supplementary Fig. S1). Our finding is consistent to a previous study on the Nav1.5 channel, which showed that the extent of block on Nav1.5 by a given LA concentration depended on the number of times the channels opened but not the stimulation frequency [40]. Moreover, the same study reported that the inhibitory effect of LA on a channel in its open state was more potent than that on a channel in its inactivated and resting states [40]. Slow-onset inhibition was also observed in a study on the inhibition of Nav1.5 mutant channels by batrachotoxin (BTX), in which the researchers proposed that the slow onset of BTX might be ascribed to its bulky size [42]. The size of LA is very similar to that of BTX (MW 584 for LA vs. 538 for BTX). Thus, we speculated that the slow-onset inhibition by LA might be ascribed to its open-channel inhibiting property and its relatively large size.

Clinical data have shown that the analgesic activity of LA may persist $8 \mathrm{~h}$ after a single systematic application in spite of the fact that its elimination half-life is less than $0.5 \mathrm{~h}[8,43]$. In contrast to the fast onset and reversible inhibition of Nav1.7 by tetracaine and bupivacaine, the inhibition of Nav1.7 by LA developed very slowly and was irreversible (Fig. $6 \mathrm{~b}$ ). Although the relationship between the long-duration analgesic effect in vivo and the slow onset or irreversibility of the LA-induced inhibition of Nav1.7 requires further investigation, these characteristics resemble those of some other irreversible drugs such as monoamine oxidase (MAO) inhibitors. Clinically used MAO inhibitors such as tranylcypromine and phenelzine are rapidly metabolized, but the inhibition of the enzyme is irreversible and thus persists for a long time after the disappearance of the prototype and its metabolites [44]. However, for local anesthetics, rapid onset and short duration are primary properties. In addition, LA did not affect channel activation or inactivation and might recognize different binding sites from local anesthetics. Therefore, we propose that LA may inhibit Nav1.7 in a different manner from local anesthetics.

Aconitine, another main alkaloid isolated from Aconitum species, is a site- 2 neurotoxin that induces severe neurological symptoms and cardiovascular collapse $[2,40]$. Due to this featured toxicity, aconitine has been widely used as an experimental tool to induce experimental tachyarrhythmias [2]. In the present study, we also evaluated the effects of aconitine on the Nav1.7 channel and found that aconitine inhibits the channel with an $\mathrm{IC}_{50}$ (with $95 \%$ confidence limits) of $59.30(52.07-66.53) \mu \mathrm{mol} / \mathrm{L}$. In contrast to the lack of effects on Nav channel activation and inactivation by LA, aconitine shifts both the channel activation and steady-state fast inactivation to more hyperpolarized potentials (Supplementary Fig. S2). Our finding is consistent to a previous study, in which aconitine inhibited the macroscopic current amplitudes of Nav1.4 and Nav1.5 channels [45].

In conclusion, we found that LA is an inhibitor of the Nav1.7 channel, a promising analgesic target predominantly distributed in the nervous system. The findings will help us to understand the featured analgesic activity of LA, thus benefiting its application in the clinic and future drug development. Since LA lacks subtype selectivity among the cardiac and neuronal isoforms, structural modifications that could improve the selectivity on neuronal isoforms and enhance its potency might be helpful to develop new analgesics with less cardiac side effects. The relationship between the long maintenance time and the binding of LA to the local anesthetic site with the irreversibility of the LA-bound channel is an interesting future topic of investigation. In contrast to other site- 2 neurotoxins, which commonly modulate Nav channel activation and inactivation [46], LA did not modify these channel properties. The identification of the determinants and mechanisms underlying this feature of the drug would be interesting.

\section{ACKNOWLEDGEMENTS}

We thank Dr. Norbert Klugbauer (Albert-Ludwigs-Universität Freiburg, Freiburg, Germany) for the gift of hNav1.7 cDNA. This work was supported by the Personalized Medicines "Molecular Signature-Based Drug Discovery and Development" (Strategic Priority Research Program of the Chinese Academy of Sciences, Grant No. XDA12040221 and XDA15050308), the National Natural Science Foundation of China (81603096, 81773707, 61327014, and 61433017), and the Shanghai Science and Technology Innovation Fund 15431901500.

\section{AUTHOR CONTRIBUTIONS}

Z-BG and YG designed and conceived the experiments; Y-FL and Y-MZ performed the experiments and contributed equally to the project; all authors analyzed the data; Y-FL, Y-MZ, and Z-BG wrote the paper.

\section{ADDITIONAL INFORMATION}

The online version of this article (https://doi.org/10.1038/s41401-018-0067-x) contains supplementary material, which is available to authorized users.

Competing interests: The authors declare no competing financial interests.

\section{REFERENCES}

1. Nyirimigabo E, Xu Y, Li Y, Wang Y, Agyemang K, Zhang Y. A review on phytochemistry, pharmacology and toxicology studies of Aconitum. J Pharm Pharmacol. 2015;67:1-19.

2. Ameri A. The effects of Aconitum alkaloids on the central nervous system. Prog Neurobiol. 1998;56:211-35.

3. Yu JZ, Zhang BJ, Jiang XT. Clinical application of Lappaconitine hydrobromide. Acad J Second Mil Med Univ. 2005;26:822-4.

4. Ono M, Satoh T. Pharmacological studies of lappaconitine. Analgesic activities. Arzneim Forsch. 1988;38:892-5.

5. Gong QA, Li M. Effect of lappaconitine on postoperative pain and serum complement 3 and 4 levels of cancer patients undergoing rectum surgery. Zhongguo Zhong Xi Yi Jie He Za Zhi. 2015;35:668-72.

6. Zheng WS. Pharmacokinetic study of lappaconitine hydrobromide transfersomes in rats by LC-MS. Pharm Anal Acta. 2011;46 (4): 432-37.

7. Chen MG, Wang QH, Lin YB. A clinical study in epidural injection with lappaconitine compound for post-operative analgesia. Chin J Integr Tradit West Med. 1996;3:257-60.

8. Huang YM, Chen XB, Wang ZS. Lappaconitine for postoperative pain: a doubleblind study. New Drugs Clin Remedies. 1997;16:75-6.

9. Sh Lu, Wang SJ. Clinical control study of lappaconitine vs morphine for postoperative epidural analgesia. Chin J New Drugs Clin Remedies. 2004;23:497-9.

10. Yang C, Liu Y, Song Z, Wang K. Clinical study of Lappaconitine for postoperative patient-controlled subcutaneous analgesia on plasma $\beta$-endorphin. China Med Herald. 2010;7:17-9.

11. Ono M, Satoh T. Pharmacological studies of lappaconitine. Occurrence of analgesic effect without opioid receptor. Res Commun Chem Pathol Pharmacol. 1989;63:13-25.

12. Guo X, Tang XC. Lappaconitine and N-deacetyllappaconitine potentiate footshock-induced analgesia in rats. Life Sci. 1991;48:1365-70.

13. Zhang L, Liu X, Wang Y, Wu J. Clinical observation of Oxy Contin combined with Lappaconitine for pain on moderate and severe chronic cancer pain. J Mod Oncol. 2016;24:2961-4.

14. Guo X, Tang XC. Effects of reserpine and 5-HT on analgesia induced by lappaconitine and N-deacetyllappaconitine. Acta Pharmacol Sin. 1990;11:14.

15. Ono M, Satoh T. Pharmacological studies on lappaconitine: possible interaction with endogenous noradrenergic and serotonergic pathways to induce antinociception. Jpn J Pharmacol. 1992;58:251-7.

16. Ou S, Zhao YD, Xiao Z, Wen HZ, Cui J, Ruan HZ. Effect of lappaconitine on neuropathic pain mediated by $\mathrm{P} 2 \mathrm{X} 3$ receptor in rat dorsal root ganglion. Neurochem Int. 2011;58:564-73.

17. Ameri A, Metzmeier $P$, Peters T. Frequency-dependent inhibition of neuronal activity by lappaconitine in normal and epileptic hippocampal slices. $\mathrm{Br} \mathrm{J}$ Pharmacol. 1996;118:577-84. 
18. Ameri A. Structure-dependent differences in the effects of the Aconitum alkaloids lappaconitine, $\mathrm{N}$-desacetyllappaconitine and lappaconidine in rat hippocampal slices. Brain Res. 1997;769:36-43.

19. Ameri A, Simmet T. Antagonism of the aconitine-induced inexcitability by the structurally related Aconitum alkaloids, lappaconitine and ajacine. Brain Res. 1999;842:332-41.

20. Valeev $A E$, Verkhratskii AN, Dzhakhangirov FN. Effects of allapinin on sodium currents in isolated neurons of the trigeminal ganglia and cardiomyocytes of rats. Biull Eksp Biol Med. 1991;111:388-90.

21. Seitz U, Ameri A. Different effects on $[3 \mathrm{H}]$ noradrenaline uptake of the Aconitum alkaloids aconitine, 3-acetylaconitine, lappaconitine, and N-desacetyllappaconitine in rat hippocampus. Biochem Pharmacol. 1998;55:883-8.

22. Catterall WA. Voltage-gated sodium channels at 60: structure, function and pathophysiology. J Physiol. 2012;590:2577-89.

23. Weng Y, Chen M, Qiu S. Curative effect observation of lappaconitine adhesive patch to radioactive stomatitis pain of patients with nasopharyngeal carcinoma. China Med Pharm. 2016;6:65-7.

24. Zhou XM, Zhong JC, Que TS, Zhang HP, Lin ZH, Wei YF, et al. Observation on clinical effect of lappaconitine adhesive patch in treatment of the pain of liver cancer. Guangxi Med J. 2005;27:1528-30.

25. Dib-Hajj SD, Yang Y, Black JA, Waxman SG. The $\mathrm{Na}(\mathrm{V}) 1.7$ sodium channel: from molecule to man. Nat Rev Neurosci. 2013;14:49-62.

26. Bennett DL, Woods CG. Painful and painless channelopathies. Lancet Neurol. 2014;13:587-99.

27. Vetter I, Deuis JR, Mueller A, Israel MR, Starobova H, Zhang A, et al. NaV1.7 as a pain target-from gene to pharmacology. Pharmacol Ther. 2017;172:73-100.

28. Cao L, McDonnell A, Nitzsche A, Alexandrou A, Saintot PP, Loucif AJ, et al. Pharmacological reversal of a pain phenotype in iPSC-derived sensory neurons and patients with inherited erythromelalgia. Sci Transl Med. 2016;8:335ra56.

29. Karoly R, Lenkey N, Juhasz AO, Vizi ES, Mike A. Fast- or slow-inactivated state preference of $\mathrm{Na}^{+}$channel inhibitors: a simulation and experimental study. PLoS Comput Biol. 2010;6:e1000818.

30. England S, de Groot MJ. Subtype-selective targeting of voltage-gated sodium channels. Br J Pharmacol. 2009;158:1413-25.

31. Bagal SK, Chapman ML, Marron BE, Prime R, Storer RI, Swain NA. Recent progress in sodium channel modulators for pain. Bioorg Med Chem Lett. 2014;24: 3690-9.
32. Ahuja S, Mukund S, Deng L, Khakh K, Chang E, Ho H, et al. Structural basis of Nav1.7 inhibition by an isoform-selective small-molecule antagonist. Science. 2015;350:aac5464.

33. Mike $A$, Lukacs $P$. The enigmatic drug binding site for sodium channel inhibitors. Curr Mol Pharmacol. 2010;3:129-44.

34. Zheng YM, Wang WF, Li YF, Yu Y, Gao ZB. Enhancing inactivation rather than reducing activation of Nav1.7 channels by a clinically effective analgesic CNV1014802. Acta Pharmacol Sin. 2017;39:587-96.

35. Cox J, Reimann F, Nicholas AK, Thornton G, Roberts E, Springell K, et al. An SCN9A channelopathy causes congenital inability to experience pain. Nature. 2006:444:894-8.

36. Nahorski MS, Chen YC, Woods CG. New Mendelian disorders of painlessness Trends Neurosci. 2015;38:712-24.

37. Goldberg YP, Price N, Namdari R, Cohen CJ, Lamers MH, Winters C, et al. Treatment of $\mathrm{Na}(\mathrm{v}) 1.7-$ mediated pain in inherited erythromelalgia using a novel sodium channel blocker. Pain. 2012;153:80-5.

38. He XH, Zang Y, Chen X, Pang RP, Xu JT, Zhou X, et al. TNF-alpha contributes to upregulation of Nav1.3 and Nav1.8 in DRG neurons following motor fiber injury. Pain. 2010;151:266-79.

39. Loussouarn G, Sternberg D, Nicole S, Marionneau C, Le Bouffant F, Toumaniantz G, et al. Physiological and pathophysiological insights of Nav1.4 and Nav1.5 comparison. Front Pharmacol. 2015;6:1-20.

40. Wright SN. Irreversible block of human heart $(\mathrm{hH} 1)$ sodium channels by the plant alkaloid lappaconitine. Mol Pharmacol. 2001;59:183-92.

41. Deuis JR, Wingerd JS, Winter Z, Durek T, Dekan Z, Sousa SR, et al. Analgesic effects of GpTx-1, PF-04856264 and CNV1014802 in a mouse model of NaV1.7-mediated pain. Toxins. 2016; 8(3): 1-19.

42. Wang SY, Tikhonov DB, Mitchell J, Zhorov BS, Wang GK. Irreversible block of cardiac mutant $\mathrm{Na}^{+}$channels by batrachotoxin. Channels. 2007;1:179-88.

43. Wang Q, Li ZJ, Sun L, Gao LY, Li MH, Hao JJ, et al. Pharmacokinetic study of lappaconitine hydrobromide in mice by LC-MS. Acta Pharmacol Sin. 2011;46:432-7.

44. Sjoqvist F. Psychotropic drugs (2). Interaction between monoamine oxidase (MAO) inhibitors and other substances. Proc R Soc Med. 1965;58:967-78.

45. Wright SN. Comparison of aconitine-modified human heart $(\mathrm{hH} 1)$ and rat skeletal (mu1) muscle $\mathrm{Na}^{+}$channels: an important role for external $\mathrm{Na}^{+}$ions. J Physiol. 2002;538:759-71.

46. Stevens M, Peigneur S, Tytgat J. Neurotoxins and their binding areas on voltagegated sodium channels. Front Pharmacol. 2011;2:71. 